\title{
MDCTA volumetric analysis for the quantification and grading of acute non-cerebral, non- gastrointestinal hemorrhage. A feasibility study.
}

\section{Stavros Spiliopoulos ( $\square$ stavspiliop@med.uoa.gr)}

National and Kapodistrian University of Athens Faculty of Medicine: Ethniko kai Kapodistriako Panepistemio Athenon latrike Schole https://orcid.org/0000-0003-1860-0568

\section{Antonios Theodosis}

National and Kapodistrian University of Athens School of Medicine: Ethniko kai Kapodistriako

Panepistemio Athenon latrike Schole

\section{Konstantinos Palialexis}

National and Kapodistrian University of Athens Faculty of Medicine: Ethniko kai Kapodistriako Panepistemio Athenon latrike Schole

\section{Evgenia Efthimiou}

National and Kapodistrian University of Athens School of Medicine: Ethniko kai Kapodistriako Panepistemio Athenon latrike Schole

\section{Lazaros Reppas}

National and Kapodistrian University of Athens School of Medicine: Ethniko kai Kapodistriako Panepistemio Athenon latrike Schole

\section{Stylianos Argentos}

National and Kapodistrian University of Athens School of Medicine: Ethniko kai Kapodistriako Panepistemio Athenon latrike Schole

\section{Dimitrios Filippiadis}

National and Kapodistrian University of Athens School of Medicine: Ethniko kai Kapodistriako Panepistemio Athenon latrike Schole

\section{Nikolaos Kelekis}

National and Kapodistrian University of Athens School of Medicine: Ethniko kai Kapodistriako Panepistemio Athenon latrike Schole

\section{Elias Brountzos}

National and Kapodistrian University of Athens School of Medicine: Ethniko kai Kapodistriako Panepistemio Athenon latrike Schole

\section{Research Article}

Keywords: Computed Tomography Angiography, Hemorrhage, Endovascular Procedures, Volumetry. 
Posted Date: March 22nd, 2021

DOI: https://doi.org/10.21203/rs.3.rs-344855/v1

License: (c) (i) This work is licensed under a Creative Commons Attribution 4.0 International License. Read Full License 


\section{Abstract}

Objectives To assess the feasibility of Multi-Detector Computed Tomography Angiography (MDCTA) volumetry for the quantification and grading of acute non-cerebral,

non-gastrointestinal bleeding.

Methods This retrospective, single-center, study investigated consecutive patients with MDCTA positive for active non-cerebral, non-gastrointestinal bleeding, between January 2020 and June 2020. Outcome measures were the quantification of active extravasation at the arterial and parenchymal phase using volumetry measurements, the calculation of active bleeding rate and bleeding grading, 30-day mortality rate, identification of independent predictors of mortality and correlation between volumetric analysis, various clinical features and the decision to proceed with an intervention.

Results In total 30 patients ( 17 females; $56.6 \%$; mean age $70.0 \pm 16.0$ years) were analyzed. Volumetric analysis was feasible in all cases resulting in excellent inter-observer variability (interclass correlation coefficient 0.999 for arterial and 0.919 for venous volume measurements). Mean volume of contrast extravasation was $1.06 \pm 1.09 \mathrm{ml}$ and $3.07 \pm 2.48 \mathrm{ml}$ at the arterial and parenchymal phases, respectively. Mean bleeding rate was $6.95 \pm 7.82 \mathrm{ml} / \mathrm{min}$. High bleeding volume at arterial phase (grade 4 bleeding) was the only independent predictor of 30-day mortality (HR 1383.58; $p=0.042)$. There was a positive correlation between bleeding volume at arterial phase $\left(r_{s}=0.340 ; p=0.033\right)$ and arterial bleeding rate $\left(r_{s}=\right.$ $0.381 ; p=0.019)$ with the decision to proceed with an intervention. Bleeding volume of $0.6 \mathrm{ml}$ was the cutoff value for the prediction of intervention (sensitivity $96.3 \%$; specificity $66.7 \%$ ).

Conclusions MDCTA volumetric analysis for the quantification and grading of acute hemorrhage was feasible with excellent inter-observer agreement. The proposed bleeding grading system could optimize decision making and predict clinical outcomes.

\section{Key Points}

- This article reports a newly proposed quantification and grading system of acute non-cerebral, nongastrointestinal hemorrhage using MDCTA volumetric analysis.

- Volumetric analysis was feasible with excellent inter-observer variability.

- For the first time, volumetric values and bleeding grading were associated with clinical outcomes and the decision to proceed to intervention.

- High bleeding volume at arterial phase (grade 4 bleeding) was the only independent predictor of 30day mortality and bleeding volume of $0.6 \mathrm{ml}$ was the cutoff value for the prediction of intervention.

\section{Introduction}


Acute hemorrhage is a medical emergency requiring a rapid multi-disciplinary therapeutic approach [1]. Hemorrhage can be either non-traumatic due to underlying pathology or traumatic, including iatrogenic and non-iatrogenic causes, while spontaneous bleeding is a distinct clinical entity, defined as the bleeding in the absence of any trauma or underlying pathology and is a possible complication of anticoagulant therapy $[2-8]$.

Radiology plays a key role in the management of acute bleeding, and multi-detector computed tomography angiography (MDCTA) has emerged as the diagnostic modality of choice, as it enables to identify the bleeding site and coexisting pathologies, with excellent sensitivity and specificity of approximately $99 \%[10,11]$. Most importantly, according to current literature, MDCTA is able to detect active bleeding rates as low as $0.3 \mathrm{~mL} / \mathrm{min}$, which is more sensitive than $0.5 \mathrm{~mL} / \mathrm{min}$ rate achieved by non-super-selective DSA $[10,11]$.

The diagnosis of active bleeding is confirmed by active contrast medium extravasation, during either dual phase or triple phase MDCTA bleeding protocols, which is identified as an extra vascular area of high attenuation. The classic imaging finding of active extravasation in MDCTA is a jet or small area of high density similar to that of the bleeding vessel during early phases, which enlarges within the hematoma during the delayed phases. [12-16] Most of the times, this finding corresponds to clinically severe bleeding and immediate intervention is required, while early recognition, characterization and localisation of bleeding is of the outmost importance, especially in hemodynamically unstable patients [14].

Treatment algorithm for bleeding includes conservative medical management such as alternating the coagulation parameters, fluid resuscitation and blood transfusion $[5,6]$, as well as invasive treatment options such as endovascular treatment (EVT) and open surgery $[6,17]$. Treatment selection should be based on a multi- disciplinary team decision, following consideration of multiple clinical and imaging parameters. The most common radiological criterion for invasive treatment is the presence of contract extravasation during the arterial phase of MDCTA. However, it is not clear whether all active extravasations noted during MDCTA should be treated invasively. Recently, the quantification of acute bleeding has been reported to offer more accurate information regarding the radiological grading of active bleeding that could be used for the optimization of the treatment algorithm, but such data are scarce and confined to abdominal and pelvic haemorrhage. [18] The authors sought to assess the feasibility and clinical implications of MDCTA volumetric analysis for the quantification and grading of acute, non-cerebral, non-gastrointestinal bleeding.

\section{Methods}

\section{Study design}

This is a retrospective, single-center, study conducted in a tertiary University Hospital. The study received approval by the Hospital's Scientific Board. The radiological images of all patients undergoing MDCTA between January 2020 and June 2020 were analyzed. Inclusion criteria were: (a) three-phases MDCTA imaging available at PACS including unenhanced, arterial and parenchymal phase, (b) active bleeding 
identified during the arterial phase. Exclusion criteria were (a) cerebral or gastrointestinal bleeding and (b) only arterial or parenchymal phase available. In total, 30 patients fulfilled the above criteria and were included in the study. Their medical records were also analyzed, and clinical and laboratory findings were recorded. The following clinical parameters were obtained from the electronic Health Record System of our Institution: Hemoglobin and Hematocrit level before the MDCTA (same day), hemodynamic instability, in-hospital mortality, therapy plan (blood transfusion, embolization, and surgery), comorbidities, sepsis, and cause of death.

\section{Volumetry protocol}

Our institution's trauma/bleeding MDCTA protocol includes 3 phases: unenhanced, arterial and parenchymal phase. All cases in our study took place in a Philips 64 Brilliance CT scanner with slice thickness of $1,5 \mathrm{~mm}$, increment $-0,75 \mathrm{~mm}$ and pitch 0,5 in all 3 phases. The parenchymal phase usually takes place approximately 60 seconds after the region of interest (ROI) threshold during arterial phase has been reached. ROI was placed at the corresponding aorta level for suspicious bleeding, for example in case of abdominal hemorrhage ROI placed in the center of abdominal aorta lumen and in case of bleeding in thorax ROI was placed in thoracic aorta respectively. Two radiologists (AT, EE) with at least 5 years' experience in Emergency-Trauma Radiology, calculated separately the extravasation rate of these bleeding cases by using Myrian ${ }^{\circledR}$ Analysis Software (Intrasense ${ }^{\circledR}$, France). In each case the amount of active extravasation volume $(\mathrm{ml})$ was calculated in arterial and parenchymal phases, by manually placing the mouse cursor and tracing active bleeding spots on each series. By clicking and coloring on each single slice the active bleeding area, the program was automatically measuring the corresponding bleeding volume, considering the slice thickness and summing up each slice' s volume. If bleeding occurred in a discontinuity manner or in different locations within the body, all bleeding volumes were added together to determine the total active bleeding volume for each patient. After correlation of the two different radiologist's results, mean arterial and parenchymal phase active bleeding volume was quantified. According to the bleeding volumetric results, bleeding rate was calculated for each case as follows: Using the bolus tracking technique at the arterial phase, the ROI was placed on the corresponding aorta level. Trigger for start of scanning was set at aortic enhancement of $150 \mathrm{HU}$. After the ROI threshold is reached, a "scan delay" occurs which is the time between bolus trigger and the helical scanning of the arterial phase. Scan delay lasts a few seconds and was detected respectively for each case. Bleeding rate was calculated based only on the active bleeding volume measured at the arterial phase using the following formula:

Active Bleeding Rate $\left(\frac{\mathrm{ml}}{\mathrm{min}}\right)=\frac{\text { Arterial phase Volume }(\mathrm{ml})}{\text { Scan Delay (seconds) } \mathrm{X} \frac{1 \text { (minute) }}{60 \text { (seconds) }}}$ 
Bleeding rate and bleeding volume were graded using a 1 to 4 system (grade 1: very low, grade 2: low, grade 3 medium and grade 4: high. The cut-off value to classify a bleeding rate as grade 4 was $\geq$ $5 \mathrm{ml} / \mathrm{min}$ which corresponds to the average volume of 1 Unit of transfused blood in one hour. The average volume of two bags of transfused blood corresponds to blood loss $>15 \%$ of total circulating blood volume which is categorized as type II bleeding [19]. Type II bleeding (15-30 \% loss of total blood volume) is the time that the patient stops being asymptomatic and correlates with the first bleeding symptoms, such as nausea, fatigue, tachycardia and tachypnea. Therefore, a severe bleeding with high bleed rate was considered as a blood loss equal or higher to 1 blood Unit per hour. Respectively, grade 3 bleeding rate was set at $\geq 3<5 \mathrm{ml} / \mathrm{min}$, grade 2 at $\geq 1<3 \mathrm{ml} / \mathrm{min}$ and grade 1 rate at $<1 \mathrm{ml} / \mathrm{min}$. The average time between trigger bolus and beginning of scanning in arterial phase (e.g. scan delay) was 10 sec, hence corresponding bleeding volumes in arterial phase were calculated, approximated and categorized in severity scale as grade $1:<0.1 \mathrm{ml}$, grade $2: \geq 0.1<0.5 \mathrm{ml}$, grade $3: \geq 0.5<1 \mathrm{ml}$, and grade $4: \geq 1 \mathrm{ml}$.

\section{Study's outcome measures}

The study's primary outcome measures were the quantification of active extravasation at the arterial and parenchymal phase of MDCTA using volumetry measurements as described above, the calculation of active bleeding rate and bleeding grading according to the volumetric analysis. The active bleeding rate was calculated by dividing the active bleeding volume by the time between bolus triggering and arterial phase.

Secondary endpoints were periprocedural (30-day) mortality rate, the identification of independent predictors of mortality and the identification of a correlation between volumetric analysis, various clinical features, and the decision to proceed with endovascular or surgical intervention.

\section{Statistical analysis}

Continuous variables were reported as mean \pm standard deviation (SD). Categorical variables were reported as absolute frequencies / percentage. Normality was evaluated initially with the KolmogorovSmirnov goodness-of-fit test and independent sample t-test and Mann-Whitney $U$ test were applied for parametric and non-parametric comparisons, respectively. The correlation between clinical variables (age, gender, $\mathrm{Hb} / \mathrm{Ht}$ values, sepsis, hemodynamic instability, anticoagulation therapy, cause of bleeding), bleeding volume, bleeding grade, bleeding rate, mortality, and treatment plan, was evaluated. Specifically, the Pearson product-moment correlation coefficient was used to assess the association between normally distributed variables and the Spearman's rank correlation coefficient was used to assess data that did not pass the normality test. A Cox multivariate regression analysis was performed for the identification of factors associated with endovascular or surgical intervention or in-hospital mortality. The variables analyzed in the multivariable model were pre-defined (based on medical relevance) and included: gender, cause of bleeding, grade of bleeding volume according to volumetric analysis at the arterial phase, grade of arterial bleeding rate, hemodynamic instability, and severe decrease of $\mathrm{Hb}(\mathrm{Hb}<7$ or $<9$ in patients with cardiac disease). Inter-observer variability was calculated for the volumetric analysis performed for the arterial and the parenchymal phases. Statistical analysis was performed with 
the IBM SPSS statistical software package (version 20.0; SPSS/PASW, Illinois, USA) and the level of statistical significance was set at $p<0.05$.

\section{Results}

In total 30 patients ( 17 females; $56.6 \%$ ) with a mean age of $70.0 \pm 16.0$ years (range: $33-88$ years) were included in the study. The main causes of hemorrhage were iatrogenic (13/30 cases; $43.3 \%)$ followed by trauma $(9 / 30$ cases; $30.0 \%$ ) and spontaneous bleeding (8/30; 26.7\%). Patients' demographical data and bleeding details are analytically reported in table 1.

Volumetric analysis was feasible in all cases and resulted in excellent inter-observer variability between the two participating independent radiologists with an interclass correlation coefficient of $0.999(95 \% \mathrm{Cl}$ 0.999-1.000) for arterial volume measurements and $0.919(95 \% \mathrm{Cl} 0.830-0.962)$ for venous volume measurements. The mean volume of contrast medium (CM) extravasation measured at the arterial phase was $1.06 \pm 1.09 \mathrm{ml}$ (range: $0.01-4.57 \mathrm{ml}$ ). The mean volume of $\mathrm{CM}$ extravasation measured at the parenchymal phase was $3.07 \pm 2.48 \mathrm{ml}$ (range: $0.06-8.92 \mathrm{ml}$ ). The volume of $\mathrm{CM}$ active extravasation increased from the arterial to the parenchymal phase in all cases. The calculated mean bleeding rate was $6.95 \pm 7.82 \mathrm{ml} / \mathrm{min}$ (range: $0.06-34.31 \mathrm{ml} / \mathrm{min}$ ) with a median scan delay time of 10 seconds (IQR: 9 $10 \mathrm{~s}$ ). The median bleeding grade was 2 (IQR: $1-3 \mathrm{ml} / \mathrm{min}$ ). Volumetric analysis with the respective estimated bleeding rates for each individual patient is analytically reported in table 2 .

Three patients were managed conservatively $(3 / 30 ; 10 \%)$, all demonstrating very low $(1 / 3$ patient; spontaneous bleeding) and low (2/3 patients; trauma bleeding) bleeding volume and bleeding rate (patients 4; 22 and 24 in table 2). In all 23 cases referred to the Interventional Radiology department, active bleeding was confirmed by digital subtraction angiography and endovascular treatment was performed. In all cases treated with open surgery bleeding was also confirmed by surgical exploration. According to Kaplan-Meier analysis, the 30 -day mortality rate was $80 \%$ (SD: $7.3 \%$; Figure 2). The six patients died due to cardiac arrest $(n=2)$, heart failure $(n=1)$, or sepsis $(n=3)$.

Multivariate analysis identified high bleeding volume at arterial phase (grade 4 bleeding) as the only independent predictor of 30-day mortality (HR 1383.58; 95\% Cl 1,298 - 1475320,736; $\mathrm{p}=0.042$ ) (Figure 3).

According to the Spearman test there was a positive correlation between bleeding volume at arterial phase $\left(r_{s}=0.340 ; N=30 ; p=0.033\right)$ and arterial bleeding rate $\left(r_{s}=0.381 ; N=30 ; p=0.019\right)$ with the decision to proceed with an endovascular or surgical intervention. According to ROC analysis a bleeding volume value of $0.6 \mathrm{mls}$ (grade 3 bleeding) was the cutoff value for the prediction of an intervention with sensitivity of $96.3 \%$ and specificity of $66.7 \%$ (area under the curve 0.864 )

\section{Discussion}

In this study, the MDCTA volumetric analysis performed for the quantification and grading of acute non cerebral, non-GI hemorrhage was feasible and accurate. Specifically, 30 consecutive bleeding events were 
measured and quantified by two independent Radiologists, with excellent inter-observer variability, both in the arterial and the parenchymal image acquisition phase. These findings confirm an analogous elegantly performed volumetric analysis of acute abdominopelvic bleeding following trauma, recently published by Borror et al. Nevertheless, there are several significant differences between these two protocols. [18] Most importantly, bleeding rate in this study was calculated based only on the volume of $\mathrm{CM}$ extravasation measured on the arterial phase and not by the previously proposed equation which based on the difference between $\mathrm{CM}$ volume measured at the parenchymal and the arterial phase. The authors have chosen to evaluate and calculate bleeding rate using only the arterial phase and consider as accurate only the volumetric analysis of the arterial phase due to the following reasons: Firstly, the increased volume of extravascular CM noted at the parenchymal phase should not be attributed only to the additional extravasation occurring during the time period between the arterial and parenchymal phase, but also to the expansion of the same $\mathrm{CM}$ in the surrounding tissues creating a significant bias in the volumetric analysis and bleeding rate calculation. Additionally, some of the $\mathrm{CM}$ could be much diluted demonstrating $\mathrm{HU}$ similar to the hematoma and the surrounding tissues, and therefore not detectable. As Borror et al acknowledge, the equation used in their study is oversimplified and a density analysis might be required to distinguish between newly extravasated and previous diluted/diffused CM. However, the feasibility of such an analysis remains unknown. Secondly, the authors noted that, in certain cases, events characterized as "large-volume" grade 3 or 4 hemorrhages, where under evaluated using the previously described equation, due to the fact that the significant $\mathrm{CM}$ volume was divided to extended time intervals taking place between the arterial and parenchymal phases (figure 4). Obviously, for different acquisition protocols performed in different centers, including more delayed parenchymal phases as to detect low-flow bleedings, bleeding rates may vary largely, whereas the use of shorter and more uniform arterial acquisition protocols could provide a more accurate bleeding rate. Moreover, volumetry based only on the arterial phase enables a faster bleeding assessment, which is crucial in the clinical setting of severe bleeding. Therefore, the authors propose the arterial phase as the basis of performing a more accurate and practical volumetric analysis. This choice was further supported by the fact that $\mathrm{CM}$ volume extravasating at the arterial phase, and not in the parenchymal phase, was the only parameter correlated with the decision to proceed with endovascular or surgical rather than conservative treatment, while high (grade 4) bleeding volume noted at arterial phase was the only independent predictor of 30-day mortality. Furthermore, this study analyzed a variety of bleeding cases, rather than pelvic trauma alone, expanding the feasibility of the method to non-traumatic events in nearly all anatomical locations. Moreover, the herein study evaluated the contribution of volumetric analysis in the therapeutic plan analyzing its correlation with subsequent intervention, a parameter that has not been previously investigated. Interestingly, in the present factors such as hemodynamic instability, sepsis, significant $\mathrm{Hb}$ decrease, where not correlated with mortality or the decision to proceed with an open surgical or endovascular procedure, as normally expected. The authors speculate that plausible explanations for these results include the 24/7 availability and long-standing experience of the surgical and interventional radiology services, that were promptly offered in nearly all cases of active bleeding $(27 / 30 ; 90.0 \%)$ with excellent hemostasis outcomes, but also the fact that a small number of cases was analyzed. The correlation between the abovementioned clinical parameters with mortality could have 
been established if a significantly larger number of bleeding cases was available. Of note, all three cases treated conservatively were graded as very low or low bleeding events with very low arterial phase bleeding volumes. Moreover, conservative treatment was successful, and the patients were discharged from the hospital, indicating a possible correlation between the proposed volumetric analysis and correct clinical decision making. Following these results, the authors intend to incorporate bleeding volumetric grading in everyday clinical practice and prospectively evaluate the effectiveness of this tool as an adjunct to the treatment algorithm of acute bleeding. Although the choice of treatment for acute hemorrhage depends on multiple clinical parameters such as $\mathrm{Ht} / \mathrm{Hb}$ decrease, hemodynamic instability, sepsis, comorbidities, bleeding site and cause, age and performance status, MDCTA imaging is today considered an indispensable tool not only for the diagnosis of hemorrhage but also for the decision of the appropriate treatment plan. In most of the cases in which active extravasation is detected in the arterial phase of MDCTA imaging, an immediate intervention is requested, as opposed to conservative treatment which is mainly considered when no active arterial extravasation is noted. However, not all cases of active extravasation require intervention, and deciding which patient would benefit from conservative treatment in the presence of a positive MDCTA remains a complicated clinical decision. Popov et al, have recently proposed imaging criteria in order to support the choice between transcatheter embolization and conservative treatment in cases of spontaneous intramuscular bleeding of the abdomen and pelvis, highlighting the necessity of radiological criteria in the management of hemorrhage. [6] Analogously, MDCTA-based volumetric analysis and bleeding grading could be added in the treatment algorithm in order to avoid overtreatment, support the correct use of invasive treatment, and set a timeframe for intervention.

Limitations of this study include the retrospective design due to which some cases might have been missed, while some data might not be available, and the single-center design not allowing the safe generalization of the results. Moreover, a small number of patients was analyzed and thus results, and more specifically the statistical findings of binary correlation and Cox multivariable analysis, should be interpreted with cautiousness. Finally, as the rate of acute bleeding should not be considered stable and is affected by numerous factors during treatment (coagulation status, transfusions, vasospasm, inotropic therapy, etc.) the proposed volumetric grading reflects just a narrow, though indicative, time frame of the bleeding event.

Conclusively, MDCTA volumetric analysis for the quantification and grading of acute non cerebral, non-GI hemorrhage was feasible and demonstrated excellent inter-observer agreement. The introduction of the proposed MDCTA-based bleeding grading system in the treatment algorithm and the quantification of CM volume extravasating at the arterial phase could optimize decision making and predict clinical outcomes. Larger prospective studies are required to validate these initial results.

\section{Abbreviations}

CM: contrast medium 
DSA: Digital Subtraction Angiography

EVT: Endovascular Treatment

HU: Hounsfield Units

Ht: Hematocrit

Hb: Hemoglobin

MDCTA: Multi-Detector Computed Tomography Angiography

RBC: Red Blood Cell

ROI: Region of Interest

\section{Declarations}

Funding: This study received no funding

Conflicts of interest/Competing interests: None

Availability of data and material: Available upon reasonable request

Code availability: Not applicable

Ethics approval: The study received approval by the Hospital's Scientific Board

Consent to participate: Not applicable

Consent for publication: Not applicable

\section{References}

1. Spahn, D.R., Bouillon, B., Cerny, V. et al.The European guideline on management of major bleeding and coagulopathy following trauma: fifth edition. Crit Care 2019; 23(1):98. https://doi.org/10.1186/s13054-019-2347-3.

2. Ioannou P, Alexakis G. Spontaneous Retroperitoneal Bleeding in a Patient with Primary Antiphospholipid Syndrome on Aspirin. Case Rep Emerg Med. 2018;2018:4397893. https://doi.org/10.1155/2018/4397893.

3. Sunga KL, Bellolio MF, Gilmore RM, Cabrera D. Spontaneous retroperitoneal hematoma: etiology, characteristics, management, and outcome. J Emerg Med. 2012; 43(2):e15761.https://doi.org/10.1016/j.jemermed.2011.06.006. 
4. Shoeb M, Fang MC. Assessing bleeding risk in patients taking anticoagulants. J Thromb Thrombolysis 2013; 35(3):312-319.https://doi.org/10.1007/s11239-013-0899-7.

5. Park SH, Lee SW, Jeon U, et al. Transcatheter arterial embolization as treatment for a life-threatening retroperitoneal hemorrhage complicating heparin therapy. Korean J Intern Med. 2011; 26(3):352355.https://doi.org/10.3904/kjim.2011.26.3.352.

6. Popov M, Sotiriadis C, Gay F, et al. Spontaneous Intramuscular Hematomas of the Abdomen and Pelvis: A New Multilevel Algorithm to Direct Transarterial Embolization and Patient Management. Cardiovasc Intervent Radiol. 2017; 40(4):537-545. https://doi.org/10.1007/s00270-017-1590-8.

7. Crowther MA, Warkentin TE. Bleeding risk and the management of bleeding complications in patients undergoing anticoagulant therapy: focus on new anticoagulant agents. Blood. 2008; 111(10):48719.https://doi.org/10.1182/blood-2007-10-120543.

8. $\mathrm{Ng} \mathrm{HJ}$, Crowther MA. New anti-thrombotic agents: emphasis on hemorrhagic complications and their management. Semin Hematol. 2006; 3(1 Suppl 1):S77S83.https://doi.org/10.1053/j.seminhematol.2005.11.023.

9. Haber ZM, Charles HW, Erinjeri JP, Deipolyi AR. Predictors of Active Extravasation and Complications after Conventional Angiography for Acute Intraabdominal Bleeding. J Clin Med. 2017; 6(4). https://doi.org/10.3390/jcm6040047.

10. Spiliopoulos S, Festas G, Theodosis A, Palialexis K, Reppas L, Konstantos C, Brountzos E. Incidence and endovascular treatment of severe spontaneous non-cerebral bleeding: a single-institution experience. Eur Radiol. 2019; 29(6):3296-3307.https://doi.org/10.1007/s00330-018-5869-1.

11. Roy-Choudhury SH, Gallacher DJ, Pilmer J, Rankin S, Fowler G, Steers J, Dourado R, Woodburn P, Adam A. Relative threshold of detection of active arterial bleeding: in vitro comparison of MDCT and digital subtraction angiography. AJR Am J Roentgenol. 2007 Nov;189(5):W23846.https://doi.org/10.2214/AJR.07.2290.

12. Melikian R, Goldberg S, Strife BJ, Halvorsen RA. Comparison of MDCT protocols in trauma patients with suspected splenic injury: superior results with protocol that includes arterial and portal venous phase imaging. Diagn Interv Radiol. 2016; 22(5):395-9.https://doi.org/10.5152/dir.2016.15232.

13. Shotar E, Soyer P, Barat M, Dautry R, Pocard M, Placé V, Camus M, Eveno C, Barret M, Dohan A. Diagnosis of acute overt gastrointestinal bleeding with CT-angiography: Comparison of the diagnostic performance of individual acquisition phases. Diagn Interv Imaging. 2017; 98(12):857863.https://doi.org/10.1016/j.diii.2017.06.018.

14. Hamilton JD, Kumaravel M, Censullo ML, Cohen AM, Kievlan DS, West OC. Multidetector CT evaluation of active extravasation in blunt abdominal and pelvic trauma patients. Radiographics. 2008; 28(6):1603-16. https://doi.org/10.1148/rg.286085522.

15. Willmann JK, Roos JE, Platz A, Pfammatter T, Hilfiker PR, Marincek B, Weishaupt D. Multidetector CT: detection of active hemorrhage in patients with blunt abdominal trauma. AJR Am J Roentgenol. 2002; 179(2):437-44. https://doi.org/10.2214/ajr.179.2.1790437. 
16. Kim SJ, Ahn SJ, Choi SJ, Park DH, Kim HS, Kim JH. Optimal CT protocol for the diagnosis of active bleeding in abdominal trauma patients. Am J Emerg Med. 2019; 37(7):13311335.https://doi.org/10.1016/j.ajem.2018.10.011.

17. Valek V, Husty J. Quality improvement guidelines for transcatheter embolization for acute gastrointestinal nonvariceal hemorrhage. Cardiovasc Intervent Radiol. 2013 Jun;36(3):60812.https://doi.org/10.1007/s00270-012-0462-5.

18. Borror W , Gaski GE , Steenburg S. Abdominopelvic bleed rate on admission CT correlates with mortality and transfusion needs in the setting of blunt pelvic fractures: a single institution pilot study. Emerg Radiol. 2019;26(1):37-44. https://doi.org/10.1007/s10140-018-1646-3.

19. Johnson AB, Burns B. Hemorrhage. [Updated 2020 Aug 10]. In: StatPearls [Internet]. Treasure Island (FL): StatPearls Publishing; 2020 Jan-. Available from: https://www.ncbi.nlm.nih.gov/books/NBK542273

\section{Tables}


Table 1. Patients' demographics and bleeding details

\begin{tabular}{c} 
Sex \\
male \\
female \\
Age (years) \\
Average Age: 70 \\
$<60$ \\
\hline $60-75$ \\
$76-85$ \\
$>85$
\end{tabular}

Patients on anticoagulant/antiplatelet therapy

Haemoglobin $(\mathrm{Hb})$ on the day of CTA $(\mathrm{g} / \mathrm{dL})$

Average Hb: 7.46

$$
\begin{aligned}
& <9 \\
& <7
\end{aligned}
$$

Haematocrit (Ht) on the day of CTA (\%)

Average Ht: 22,05

$$
\begin{aligned}
& <25 \\
& <20
\end{aligned}
$$

Haemodynamic unstable

\section{Bleeding Causes}

Iatrogenic (placing CVL, post-operative, post- biopsy/ puncture or other interventional procedure)

$$
\text { Trauma }
$$

Spontaneous

\section{Anatomic location of haematoma}

$$
\begin{gathered}
\text { Retroperitoneal } \\
\text { Rectus Sheath } \\
\text { Pelvic }
\end{gathered}
$$

\section{Femoral}

Axillary / lateral thoracic wall

Intrahepatic with haemoperitoneum

$$
\text { Supraclavicular }
$$




\begin{tabular}{|c|c|}
\hline Bleeding vessel* & \\
\hline Lumbar Artery & 6 \\
\hline Inferior Epigastric Artery & 5 \\
\hline Internal Iliac Artery & 5 \\
\hline Deep Femoral Artery & 4 \\
\hline Renal (or polar) Artery & 3 \\
\hline Circumflex Iliac Artery & 2 \\
\hline Axillary Artery & 1 \\
\hline Subclavian Artery & 1 \\
\hline Internal Mammary Artery & 1 \\
\hline Hepatic Artery & 1 \\
\hline Superior Mesenteric Artery & 1 \\
\hline Sepsis & 5 \\
\hline Therapy chosen & \\
\hline Surgery & 4 \\
\hline EVT (embolization or stent graft or both) & 23 \\
\hline Conservative Treatment & 3 \\
\hline Deaths & \\
\hline Overall & 6 \\
\hline Directly bleeding- related & 0 \\
\hline $\begin{array}{l}\text { hemoglobin; Ht: hematocrit; CVL: central venous line; E } \\
\text { cases of intervention this was confirmed by either angio }\end{array}$ & \\
\hline
\end{tabular}




\begin{tabular}{|c|c|c|c|c|c|}
\hline & \multicolumn{2}{|c|}{ Rad 1} & \multicolumn{2}{|c|}{ Rad 2} & \\
\hline Patient No & Arteria & se (ml) & $\overline{\text { Delayec }}$ & se (ml) & $\begin{array}{c}\text { Active bleeding rate } \\
(\mathrm{ml} / \mathrm{min})\end{array}$ \\
\hline 1 & 1,83 & 1,81 & 9,01 & 8,9 & 12,13 \\
\hline 2 & 0,26 & 0,25 & 1,72 & 1,74 & 1,53 \\
\hline 3 & 0,81 & 0,82 & 4,71 & 4,8 & 4,89 \\
\hline 4 & 0,01 & 0,01 & 0,05 & 0,07 & 0,06 \\
\hline 5 & 2,18 & 2,15 & 2,92 & 2,94 & 12,99 \\
\hline 6 & 1,67 & 1,65 & 6,4 & 6,43 & 9,96 \\
\hline 7 & 0,36 & 0,35 & 1,09 & 1,11 & 2,37 \\
\hline 8 & 0,8 & 0,78 & 2,3 & 2,32 & 4,74 \\
\hline 9 & 2,5 & 2,52 & 5,67 & 5,7 & 16,73 \\
\hline 10 & 2,5 & 2,5 & 6,98 & 7,01 & 15,00 \\
\hline 11 & 0,32 & 0,31 & 2,56 & 2,58 & 2,10 \\
\hline 12 & 0,08 & 0,07 & 0,68 & 0,7 & 0,45 \\
\hline 13 & 2,7 & 2,62 & 5,26 & 5,44 & 17,73 \\
\hline 14 & 0,9 & 0,92 & 2,77 & 2,79 & 4,96 \\
\hline 15 & 0,61 & 0,63 & 2,63 & 2,65 & 4,13 \\
\hline 16 & 0,04 & 0,05 & 1,13 & 1,14 & 0,27 \\
\hline 17 & 0,6 & 0,62 & 2,24 & 2,27 & 4,58 \\
\hline 18 & 0,09 & 0,11 & 0,66 & 0,68 & 0,60 \\
\hline 19 & 0,07 & 0,08 & 2,88 & 2,91 & 0,45 \\
\hline 20 & 0,3 & 0,28 & 0,38 & 0,4 & 1,74 \\
\hline 21 & 1,2 & 1,23 & 6 & 6,12 & 7,29 \\
\hline 22 & 0,29 & 0,26 & 0,53 & 0,56 & 1,83 \\
\hline 23 & 0,51 & 0,53 & 2,02 & 2,1 & 3,47 \\
\hline 24 & 0,28 & 0,27 & 0,3 & 0,31 & 1,83 \\
\hline 25 & 2,35 & 2,28 & 3,33 & 3,2 & 12,63 \\
\hline 26 & 0,53 & 0,59 & 1,3 & 1,32 & 4,80 \\
\hline 27 & 4,7 & 4,45 & 6,1 & 6,15 & 34,31 \\
\hline 28 & 0,26 & 0,29 & 0,84 & 0,91 & 1,18 \\
\hline 29 & 2,38 & 2,4 & 7,7 & 7,5 & 20,49 \\
\hline 30 & 0,85 & 0,9 & 1,58 & 1,65 & 3,28 \\
\hline
\end{tabular}

\section{Figures}




\section{Number of Active Bleeding Sites}

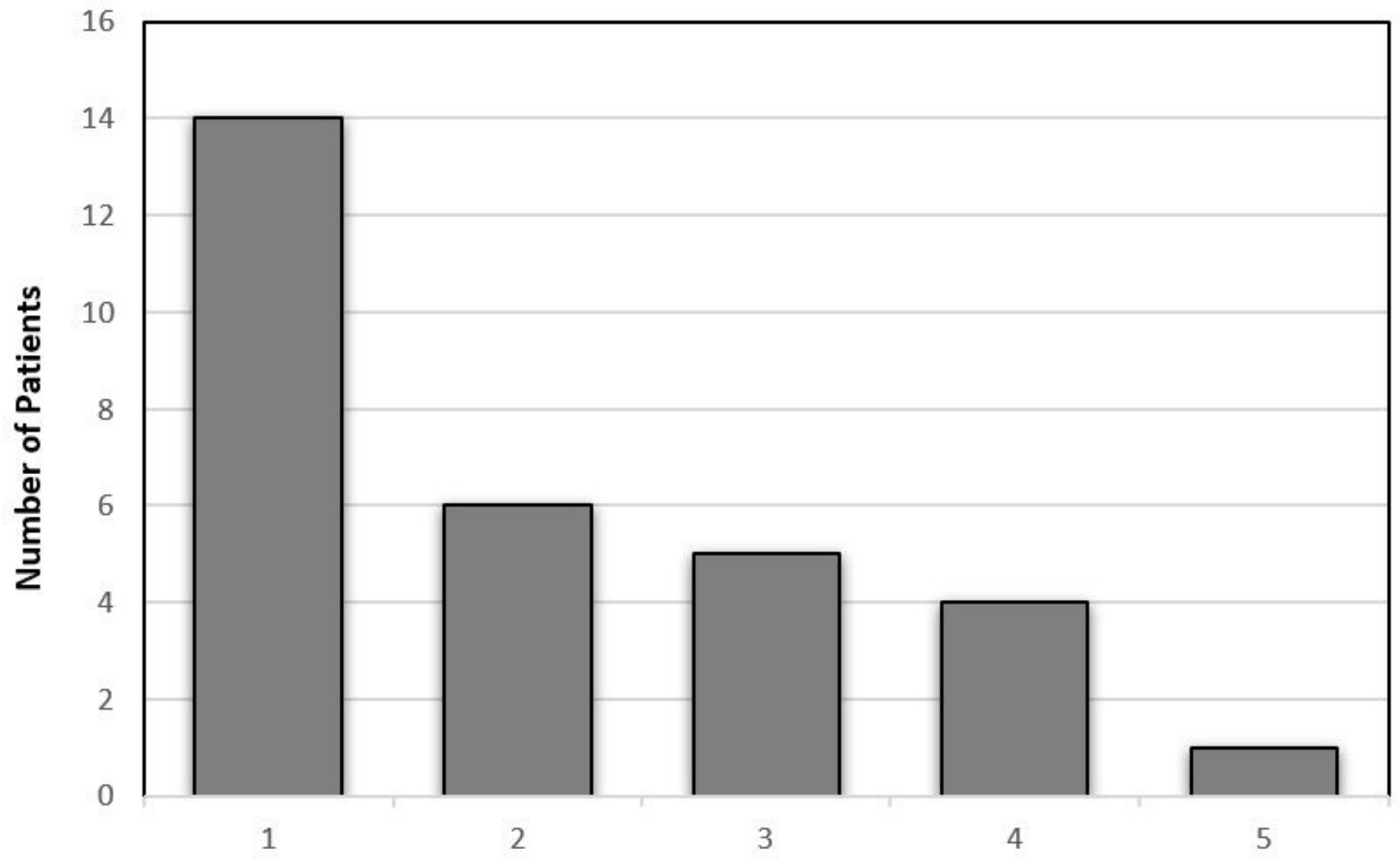

Figure 1

Number of the analyzed bleeding sites per patient. 


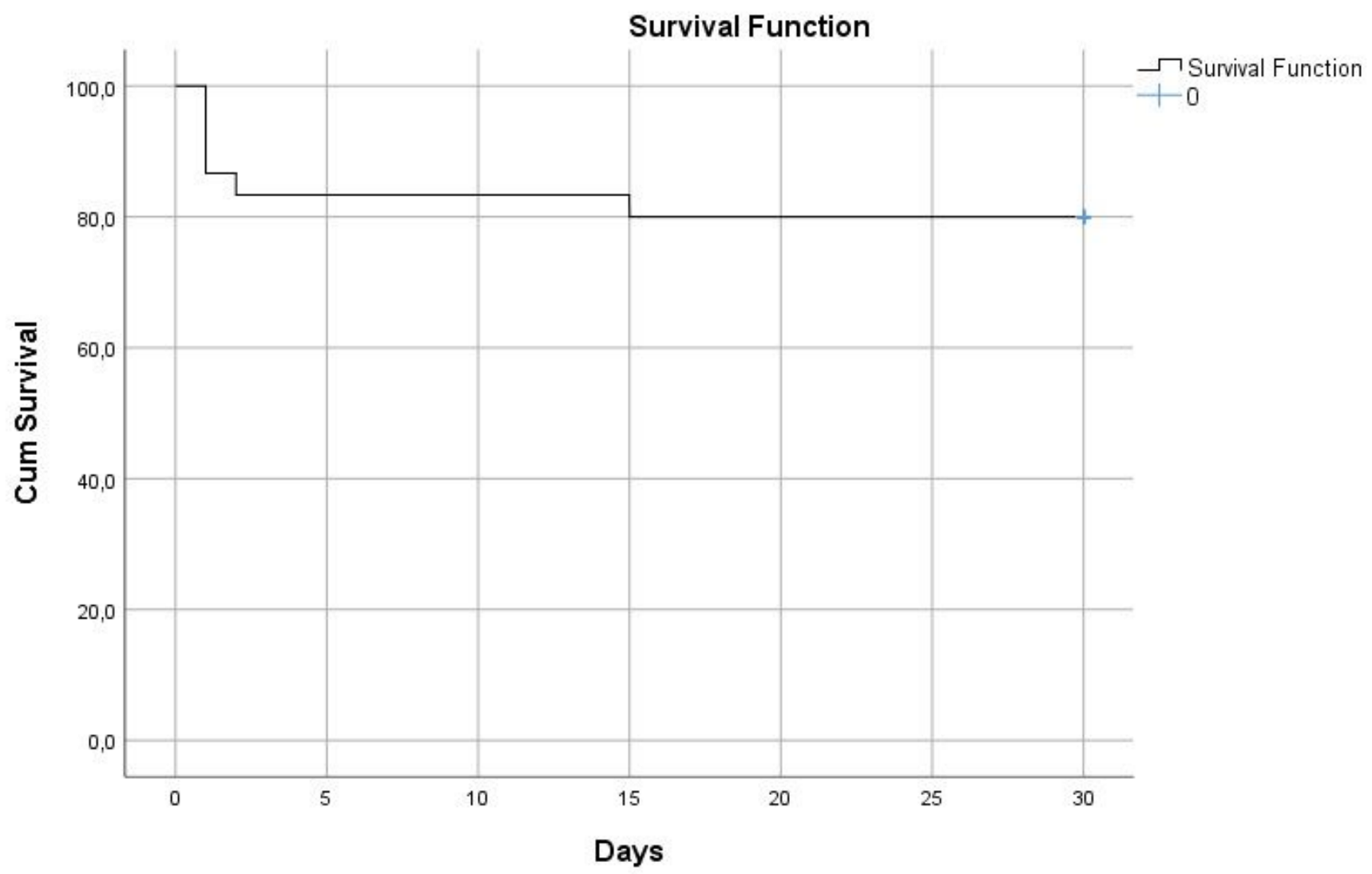

Figure 2

Kaplan-Meir plot of 30-day patients' survival. 


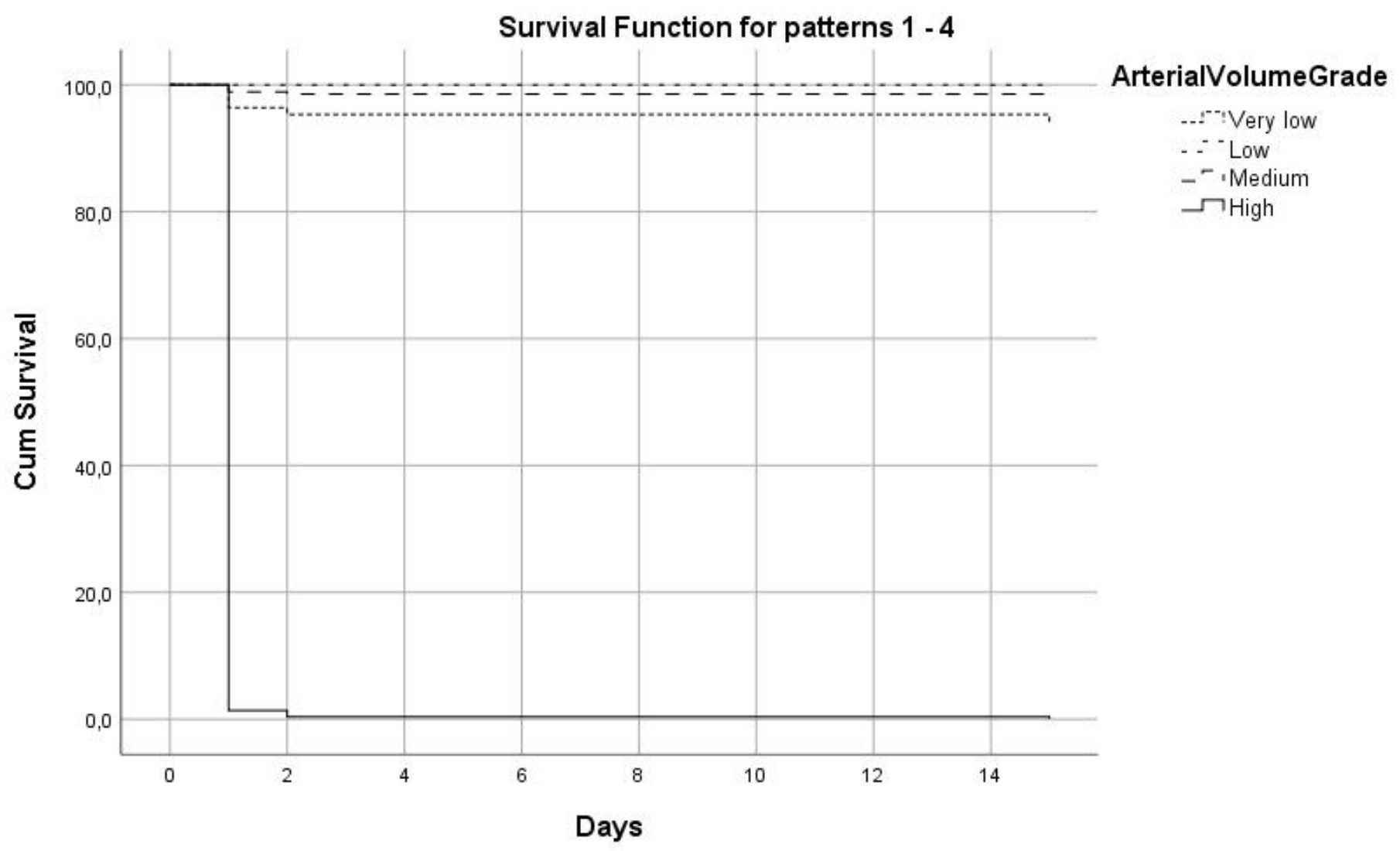

Figure 3

Cox multivariable analysis plot of 30-days patient survival stratified according to bleeding volume grade. 


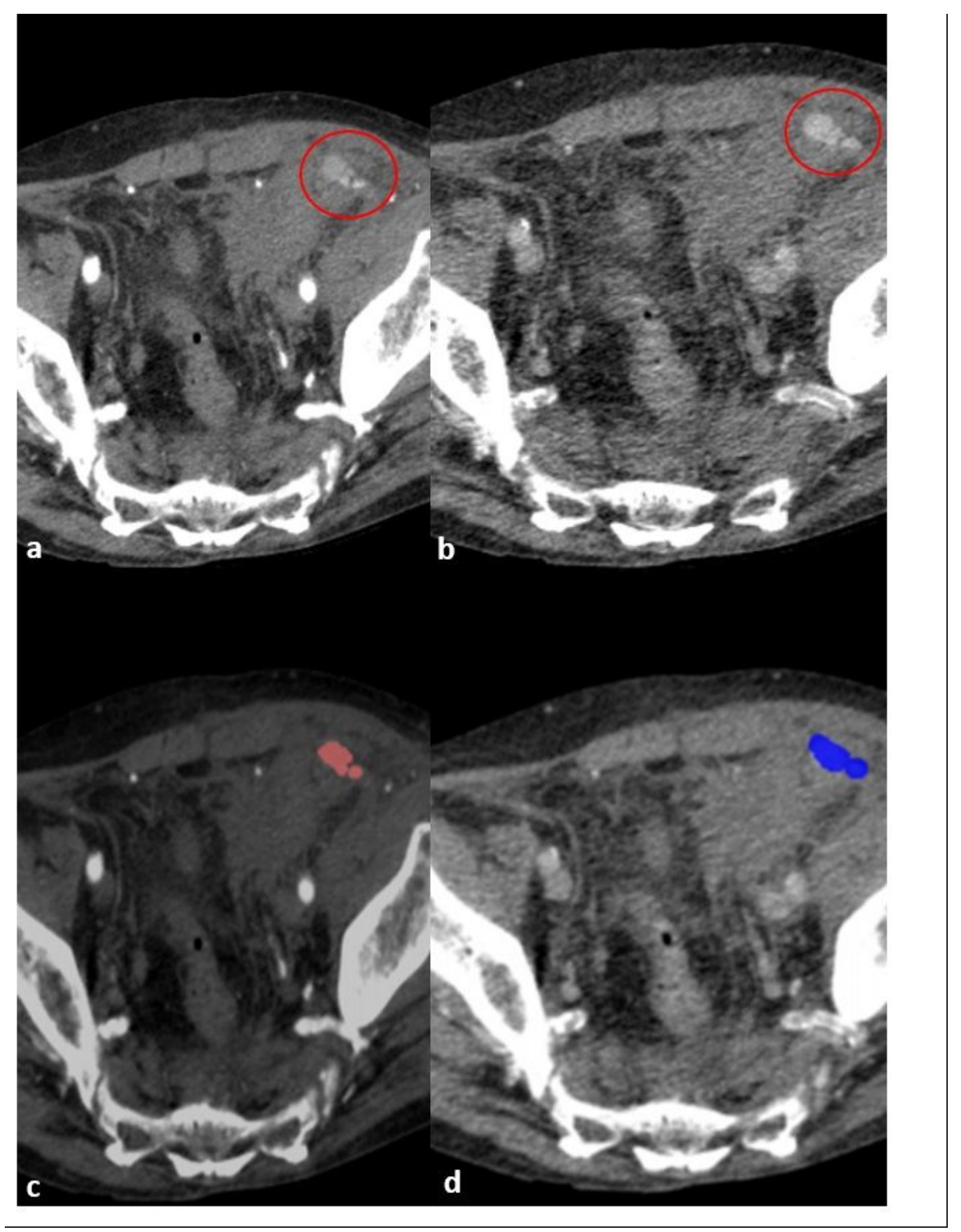

\section{Figure 4}

Patient with huge left retroperitoneal hematoma and active bleeding from CFA. The patient suffered from clinically severe bleeding (grade 3; hemodynamically unstable with $\mathrm{Hb}$ : 6.5). Axial CTA image depicting active bleeding during arterial (circle in image a) and parenchymal (circle in image b) phase. Images $\mathrm{C}$ and $\mathrm{D}$ showing the corresponding active extravasation volume measurements in each phase. The mean active bleeding volume was calculated $2.66 \mathrm{ml}$ and $5.35 \mathrm{ml}$ respectively for arterial and parenchymal 
phases. Using the previously proposed formula [18]: Bleeding Rate $(\mathrm{ml} / \mathrm{min})=(5.35 \mathrm{ml}-2.66 \mathrm{ml}) /$

[(10:45:24 PM- 10:44:02 PM) X (1 minute) / (60 seconds)] $=(2.69 \mathrm{ml}) /(82$ seconds $X[(1$ minute $) /(60$

seconds)] $=1.97 \mathrm{ml} / \mathrm{min}$ Using the formula proposed in this study: Bleeding Rate $(\mathrm{ml} / \mathrm{min})=(2.66 \mathrm{ml}) /(9$ seconds) $X[(1$ minute) $/$ ( 60 seconds $)]=17.73 \mathrm{ml} / \mathrm{min}$. 